\title{
$P$-partitions and a multi-parameter Klyachko idempotent
}

\author{
Peter McNamara \\ Instituto Superior Técnico \\ Departamento de Matemática \\ Avenida Rovisco Pais \\ 1049-001 Lisboa, Portugal \\ mcnamara@math.ist.utl.pt
}

Christophe Reutenauer

Laboratoire de Combinatoire et d'Informatique Mathématique

Université du Québec à Montréal

Case Postale 8888, succursale Centre-ville

Montréal (Québec) H3C 3P8, Canada

christo@lacim.uqam.ca

Submitted: Jun 30, 2005; Accepted: Oct 24, 2005; Published: Oct 31, 2005

Mathematics Subject Classifications: 17B01, 05E99, 05A30

Dedicated to Richard Stanley on the occasion of his 60th birthday

\begin{abstract}
Because they play a role in our understanding of the symmetric group algebra, Lie idempotents have received considerable attention. The Klyachko idempotent has attracted interest from combinatorialists, partly because its definition involves the major index of permutations.

For the symmetric group $S_{n}$, we look at the symmetric group algebra with coefficients from the field of rational functions in $n$ variables $q_{1}, \ldots, q_{n}$. In this setting, we can define an $n$-parameter generalization of the Klyachko idempotent, and we show it is a Lie idempotent in the appropriate sense. Somewhat surprisingly, our proof that it is a Lie element emerges from Stanley's theory of $P$-partitions.
\end{abstract}




\section{Introduction}

The motivation for our work is centered around the search for Lie idempotents in the symmetric group algebra. In fact, our goal is to give a generalization of the well-known Klyachko idempotent, and to show that important and interesting properties of the Klyachko idempotent carry over to the extended setting. It turns out that the proof that our generalized Klyachko idempotent is a Lie element gives a nice application and illustration of Richard Stanley's theory of $P$-partitions. We should point out that $P$-partitions were previously used in [2] to show that the traditional Klyachko idempotent is a Lie element.

To define Lie idempotents, however, we will first need the concepts of free Lie algebras and the symmetric group algebra. Let $K$ be a field of characteristic 0 . If $X$ is an alphabet, we will write $K\langle X\rangle$ to denote the free associative algebra consisting of all linear combinations of words on $X$ with coefficients in $K$. The product of two words on $X$ is defined to be their concatenation, and extending this product by linearity gives a product on $K\langle X\rangle$. We can then define the Lie bracket $[p, q]$ of two elements $p$ and $q$ of $K\langle X\rangle$ by $[p, q]=p q-q p$. We let $\mathcal{L}_{K}(X)$ denote the smallest vector subspace of $K\langle X\rangle$ containing $X$ and closed under the Lie bracket. It is a classical result that $\mathcal{L}_{K}(X)$ is the free Lie algebra on $X$. We refer the reader to [6, 16] for further details on free Lie algebras from a combinatorial viewpoint.

If $X=\{1,2, \ldots, n\}$, then elements of the symmetric group $S_{n}$ can be considered as words on $X$. We write $K S_{n}$ to denote the symmetric group algebra, which consists of linear combinations of elements of the symmetric group $S_{n}$, with coefficients in $K$. When $X=$ $\{1,2, \ldots, n\}$, certain elements of $\mathcal{L}_{K}(X)$, such as $[[\cdots[1,2], 3], \ldots, n]$, can be naturally considered to be elements of $K S_{n}$. This is because all the words in their expansions as elements of $K\langle X\rangle$ are permutations of $1,2, \ldots, n$. Elements in this intersection of $K S_{n}$ and $\mathcal{L}_{K}(X)$ are called Lie elements. We will denote the set of Lie elements by $\mathcal{L}_{n}$.

We should clarify our suggestion that $K S_{n}$ is an algebra. The product of two permutations $\sigma, \tau \in S_{n}$ is the usual composition $\sigma \tau$ from right to left, and extending this product by linearity gives the product in $K S_{n}$. It is well-known, and is not difficult to check, that $\mathcal{L}_{n}$ is then a left ideal of $K S_{n}$.

Definition 1.1. A Lie idempotent is an element $\pi$ of $K S_{n}$ that is idempotent and that satisfies

$$
K S_{n} \pi=\mathcal{L}_{n}
$$

In particular, $\pi$ must be a Lie element. Lie idempotents are quite remarkable because, in particular, they give an alternative, and direct, construction of $\mathcal{L}_{n}$. It is natural, therefore, that there should be widespread interest in the search for Lie idempotents and, from a combinatorial perspective, [2, 4, 7, 6, 11, 15] all offer progress in this search.

One of the most famous Lie idempotents is the Klyachko idempotent of [10]. (We refer the reader to the end of this introduction for the definition of the major index, $\operatorname{maj}(\sigma)$, of $\sigma \in S_{n}$.) Let $\zeta$ be a primitive $n$th root of unity in $K$, meaning that $\zeta^{n}=1$ and $\zeta^{m} \neq 1$ 
for $1 \leq m<n$. Then the Klyachko idempotent $\kappa_{n}$ is defined by

$$
\kappa_{n}=\frac{1}{n} \sum_{\sigma \in S_{n}} \zeta^{\operatorname{maj}(\sigma)} \sigma .
$$

The appearance of the major index in this definition naturally makes the Klyachko idempotent appealing to combinatorialists and, for example, [2, 4, 11, 15] study the Klyachko idempotent and its generalizations.

Our goal is to introduce a new, broad generalization of the Klyachko idempotent and to show that its Lie idempotency property is preserved in this much wider setting. As we will show in Example 1.6, we will indeed be able to recover the usual symmetric group algebra $K S_{n}$ and the Klyachko idempotent by specialization. Rather than working with a primitive $n$th root of unity, we will let $\mathbf{q}=\left(q_{1}, q_{2}, \ldots, q_{n}\right)$ be a sequence of variables in a field $K$ with the only restriction being that $q_{1} q_{2} \cdots q_{n}=1$. Since the $q_{i}$ 's are formal variables, we can assume, in particular, that $q_{i_{1}} q_{i_{2}} \cdots q_{i_{r}} \neq 1$ for any proper subset $\left\{i_{1}, i_{2}, \ldots, i_{r}\right\}$ of $\{1,2, \ldots, n\}$. Throughout, unless otherwise stated, $\mathbf{q}$ will denote such a sequence. We let $K(\mathbf{q})$ denote the field of rational functions in $\mathbf{q}$ over the field $K$, and our primary focus will be $K(\mathbf{q}) S_{n}$, the symmetric group algebra with coefficients in $K(\mathbf{q})$. Before proceeding, however, we must pay attention to a twist in our story. It turns out that the most useful product for $K(\mathbf{q}) S_{n}$ is not the natural analogue of the product for $K S_{n}$. More precisely, if $f(\mathbf{q}), g(\mathbf{q}) \in K(\mathbf{q})$ and $\sigma, \tau \in S_{n}$, one might assume that the product of $f(\mathbf{q}) \sigma$ and $g(\mathbf{q}) \tau$ should be defined to be simply $(f(\mathbf{q}) g(\mathbf{q})) \sigma \tau$. However, this product does not seem to allow the concepts of interest from $K S_{n}$ to extend to $K(\mathbf{q}) S_{n}$ and, in particular, our generalized Klyachko element is not idempotent with respect to this product for $n \geq 4$. Instead, we observe that there is a natural left action of $S_{n}$ on $K(\mathbf{q})$ : if $f(\mathbf{q})=f\left(q_{1}, q_{2}, \ldots, q_{n}\right) \in K(\mathbf{q})$, then we define

$$
\sigma[f(\mathbf{q})]=f\left(q_{\sigma(1)}, q_{\sigma(2)}, \ldots, q_{\sigma(n)}\right) .
$$

We then define the twisted product of $f(\mathbf{q}) \sigma$ and $g(\mathbf{q}) \tau$, denoted $f(\mathbf{q}) \sigma \ltimes g(\mathbf{q}) \tau$, by

$$
f(\mathbf{q}) \sigma \ltimes g(\mathbf{q}) \tau=(f(\mathbf{q}) \sigma[g(\mathbf{q})]) \sigma \tau .
$$

As a simple example, if $n=3$,

$$
231 \ltimes \frac{q_{1} q_{3}}{\left(1-q_{1}\right)\left(1-q_{1} q_{3}\right)} 132=\frac{q_{2} q_{1}}{\left(1-q_{2}\right)\left(1-q_{2} q_{1}\right)} 213 .
$$

This twisted product appears in some standard texts on the representation theory of groups and algebras, such as [3, §28]. As in [3, we leave it as a quick exercise to check that the twisted product is associative. Our results will serve as evidence in favor of the assertion that the twisted product is the "correct" product for $K(\mathbf{q}) S_{n}$.

We are now in a position to define our extended version of the Klyachko idempotent.

Definition 1.2. Given a permutation $\sigma$ in $S_{n}$, define the $\mathbf{q}$-major index $\operatorname{maj}_{\mathbf{q}}(\sigma)$ of $\sigma$ by

$$
\operatorname{maj}_{\mathbf{q}}(\sigma)=\frac{\prod_{j \in D(\sigma)} q_{\sigma(1)} q_{\sigma(2)} \cdots q_{\sigma(j)}}{\prod_{i=1}^{n-1}\left(1-q_{\sigma(1)} q_{\sigma(2)} \cdots q_{\sigma(i)}\right)}
$$


We will justify the terminology "q-major index" in Example 1.6.

Remark 1.3. The numerator terms $N_{\mathbf{q}}(\sigma)=\prod_{j \in D(\sigma)} q_{\sigma(1)} \ldots q_{\sigma(j)}$ have a certain fame due to their appearance in [5]. There, Garsia shows that every polynomial $G(\mathbf{q})$ in $q_{1}, \ldots, q_{n}$ has a unique expression of the form

$$
G(\mathbf{q})=\sum_{\sigma \in S_{n}} g_{\sigma}(\mathbf{q}) N_{\mathbf{q}}(\sigma)
$$

where each $g_{\sigma}(\mathbf{q})$ is a polynomial that is symmetric in $q_{1}, \ldots, q_{n}$. Furthermore, if $G(\mathbf{q})$ has integer coefficients, then so do all the polynomials $g_{\sigma}(\mathbf{q})$. These results were originally conjectured by Ira Gessel.

The following definition introduces our main object of study.

Definition 1.4. Denote by $\kappa_{n}(\mathbf{q})$ the element of $K(\mathbf{q}) S_{n}$ given by

$$
\kappa_{n}(\mathbf{q})=\sum_{\sigma \in S_{n}} \operatorname{maj}_{\mathbf{q}}(\sigma) \sigma
$$

Example 1.5. If $n=3$ we get

$$
\begin{aligned}
\kappa_{3}(\mathbf{q})= & \frac{1}{\left(1-q_{1}\right)\left(1-q_{1} q_{2}\right)} 123+\frac{q_{1} q_{3}}{\left(1-q_{1}\right)\left(1-q_{1} q_{3}\right)} 132 \\
& +\frac{q_{2}}{\left(1-q_{2}\right)\left(1-q_{1} q_{2}\right)} 213+\frac{q_{2} q_{3}}{\left(1-q_{2}\right)\left(1-q_{2} q_{3}\right)} 231 \\
& +\frac{q_{3}}{\left(1-q_{3}\right)\left(1-q_{1} q_{3}\right)} 312+\frac{q_{2} q_{3}^{2}}{\left(1-q_{3}\right)\left(1-q_{2} q_{3}\right)} 321 .
\end{aligned}
$$

Example 1.6. With $\zeta$ a primitive $n$th root of unity, we can see that $\kappa_{n}(\mathbf{q})$ maps to the Klyachko idempotent $\kappa_{n}$ under the specialization $q_{i} \rightarrow \zeta$ for $i=1, \ldots, n$. Indeed, the q-major index of any $\sigma \in S_{n}$ then specializes to

$$
\frac{\zeta^{\mathrm{maj}(\sigma)}}{\prod_{i=1}^{n-1}\left(1-\zeta^{i}\right)}=\frac{\zeta^{\mathrm{maj}(\sigma)}}{n} .
$$

This equality follows from the identity

$$
x^{n}-1=(x-1)(x-\zeta)\left(x-\zeta^{2}\right) \cdots\left(x-\zeta^{n-1}\right),
$$

which implies that $n=(1-\zeta)\left(1-\zeta^{2}\right) \cdots\left(1-\zeta^{n-1}\right)$. Also notice that, since $q_{1}=\cdots=q_{n}$, the twisted product of $K(\mathbf{q}) S_{n}$ in this setting is identical to the usual product of $K S_{n}$.

Actually, if we take the ring $K[q]$, localized at $1-q, \ldots, 1-q^{n-1}$, and quotiented by the ideal generated by $1-q^{n}$, it will follow from our results that the element

$$
\sum_{\sigma \in S_{n}} \frac{q^{\operatorname{maj}(\sigma)}}{(1-q) \cdots\left(1-q^{n-1}\right)} \sigma
$$

is a Lie idempotent. This may shed some light on some results in [2]. We see that this new element specializes to the Klyachko idempotent when we map $q$ to a primitive root of unity. 
We can now state our main results.

Theorem 1.7. $\kappa_{n}(\mathbf{q})$ is a Lie element.

Theorem 1.8. $\kappa_{n}(\mathbf{q}) \ltimes \kappa_{n}(\mathbf{q})=\kappa_{n}(\mathbf{q})$, i.e., $\kappa_{n}(\mathbf{q})$ is idempotent as an element of $K(\mathbf{q}) S_{n}$.

We will let $\mathcal{L}_{n}(\mathbf{q})$ denote the analogue of $\mathcal{L}_{n}$ when coefficients come from $K(\mathbf{q})$. As a formula, $\mathcal{L}_{n}(\mathbf{q})=K(\mathbf{q}) S_{n} \cap \mathcal{L}_{K(\mathbf{q})}(\{1,2, \ldots, n\})$. We observe that $\mathcal{L}_{n}(\mathbf{q})$ is a left ideal of $K(\mathbf{q}) S_{n}$ :

$$
K(\mathbf{q}) S_{n} \ltimes \mathcal{L}_{n}(\mathbf{q})=\mathcal{L}_{n}(\mathbf{q}) .
$$

Theorem 1.9. The left ideal $K(\mathbf{q}) S_{n} \ltimes \kappa_{n}(\mathbf{q})$ is equal to $\mathcal{L}_{n}(\mathbf{q})$.

By extending Definition 1.1 in the obvious way, we define what it means for an element of $K(\mathbf{q}) S_{n}$ to be a Lie idempotent, and we have the following immediate consequence of Theorems 1.8 and 1.9

Corollary 1.10. $\kappa_{n}(\mathbf{q})$ is a Lie idempotent.

One might wonder why $\kappa_{n}(\mathbf{q})$ would have such desirable properties. In Section 5, we give one possible explanation. Removing the condition that $q_{1} \cdots q_{n}=1$, we consider an expression $\Theta(\mathbf{q})$, which one can think of as a generating function for $\kappa_{n}(\mathbf{q})$, defined by

$$
\Theta(\mathbf{q})=\sum_{n \geq 0} \frac{\kappa_{n}\left(q_{1}, \ldots, q_{n}\right)}{\left(1-q_{1} \cdots q_{n}\right)} .
$$

As our main result of Section 5, we show that $\Theta(\mathbf{q})$ can be expressed as a very simple infinite product. This result generalizes [8, Proposition 5.10], which corresponds to the specialization $q_{i} \rightarrow q$ for $i=1, \ldots, n$.

Remark 1.11. According to the referee, our work possibly has a generalization in the spirit of the papers of Lascoux, Leclerc and Thibon [12], and of Hivert [9]. In [12], a multiparameter construction is devised, not for the Klyachko idempotent, but for rectangularshaped $q$-Kostka numbers. On the other hand, $q$-Kostka numbers are defined in terms of Hall-Littlewood polynomials, and [9] shows a direct connection between column-shaped Hall-Littlewood polynomials and the Klyachko idempotent.

The organization of the remainder of the paper is simple: in Sections 2, 3 and 4, we prove Theorems 1.7, 1.8 and 1.9 respectively. The infinite product expansion is the subject of Section 5 .

Before beginning the proofs, we need to introduce some terminology related to permutations. If $w$ is a word of length $n$, we will write $w(i)$ to denote the $i$ th letter of $w$. If the letters of $w$ are distinct, we define the descent set $D(w)$ of $w$ by $D(w)=\{i \mid 1 \leq i \leq n-1, w(i)>w(i+1)\}$. The major index maj $(w)$ of $w$ is then the sum of the elements of $D(w)$. We will denote the cardinality of $D(w)$ by $d(w)$. Finally, we will use $\bar{D}(w)$ to denote the of circular descent set of $w$, so that

$$
\bar{D}(w)=\left\{\begin{array}{ll}
D(w) & \text { if } w(n)<w(1) \\
D(w)+\{n\} & \text { if } w(n)>w(1)
\end{array} .\right.
$$

Then $\bar{d}(w)$ is simply the cardinality of $\bar{D}(w)$. 


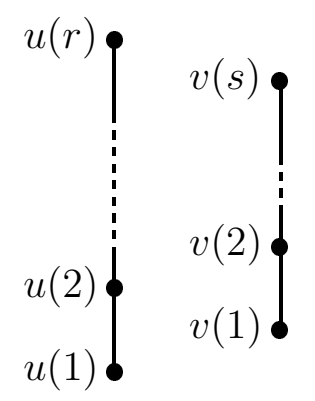

Figure 1: $P_{u, v}$

\section{$2 \kappa_{n}(\mathbf{q})$ is a Lie element}

Our goal for this section is to prove Theorem [1.7 We begin by stating a well-known characterization of Lie elements. We refer the reader to [6], [13, p. 87] or [16, $\S \S 1.3-1.4]$ for further details.

Our definitions in this paragraph will hold for $\mathbb{K}$ being any field of characteristic 0 , although we will only need them for the case $\mathbb{K}=K(\mathbf{q})$. First, define an inner (scalar) product $\langle$,$\rangle on \mathbb{K}\langle X\rangle$ by $\langle u, v\rangle=\delta_{u, v}$ for any words $u$ and $v$, extended to $\mathbb{K}\langle X\rangle$ by linearity. For the remainder of this section, it suffices to restrict to the case when $X=\{1,2, \ldots, n\}$. Suppose $u=u(1) u(2) \ldots u(r)$ and $v=v(1) v(2) \ldots v(s)$ and, since it will be sufficiently general for our needs, assume $u(i) \neq v(j)$ for all $i, j$. A word $w$ is said to be a shuffle of $u$ and $v$ if $w$ has length $r+s$ and if $u$ and $v$ are both subsequences of $w$. The shuffle product $u ш v$ of $u$ and $v$ is an element of $\mathbb{K}\langle X\rangle$ and is defined to be the sum of all the shuffles of $u$ and $v$. We will write $w \in u ш v$ if $w$ is a shuffle of $u$ and $v$. The characterization of Lie elements that we will use is the following: an element $p$ of $\mathbb{K} S_{n}$ is a Lie element if and only if $p$ is orthogonal to $u ш v$ for all non-empty words $u$ and $v$. Therefore, we wish to show that

$$
\left\langle\kappa_{n}(\mathbf{q}), u \amalg v\right\rangle=0
$$

for all $u=u(1) u(2) \cdots u(r)$ and $v=v(1) v(2) \cdots v(s)$ with $r, s \geq 1$.

Because (2.1) holds trivially otherwise, let us assume that $r+s=n$ and that $u(1), u(2), \ldots, u(r), v(1), v(2), \ldots, v(s)$ are all distinct. Therefore, the partially ordered set (poset) $P_{u, v}$ whose Hasse diagram is shown in Figure 1 is a poset with elements $\{1,2, \ldots, n\}$. Namely, $P_{u, v}$ is the disjoint union of the chains $u(1)<u(2)<\cdots<u(r)$ and $v(1)<v(2)<\cdots<v(s)$. Recall that a linear extension $\sigma$ of a poset $P$ of size $n$ is a bijection $\sigma: P \rightarrow\{1,2, \ldots, n\}$ such that if $y \leq z$ in $P$, then $\sigma(y) \leq \sigma(z)$. We will represent the linear extension $\sigma$ as the word $\sigma^{-1}(1), \sigma^{-1}(2), \ldots, \sigma^{-1}(n)$, and we will write $L(P)$ to denote the set of linear extensions of $P$. We introduce linear extensions and the poset $P_{u, v}$ for the following reason: the set of shuffles of $u$ and $v$ is exactly the set of linear 
extensions of $P_{u, v}$. Therefore, $\left\langle\kappa_{n}(\mathbf{q}), u ш v\right\rangle$ can be expressed as

$$
\left\langle\kappa_{n}(\mathbf{q}), u ш v\right\rangle=\sum_{\sigma \in L\left(P_{u, v}\right)} \frac{\prod_{j \in D(\sigma)} q_{\sigma(1)} q_{\sigma(2)} \cdots q_{\sigma(j)}}{\prod_{i=1}^{n-1}\left(1-q_{\sigma(1)} q_{\sigma(2)} \ldots q_{\sigma(i)}\right)} .
$$

The reader who is acquainted with Richard Stanley's theory of $P$-partitions may find (2.2) strikingly familiar. We now introduce the parts of this theory that will be necessary to complete our proof. While $P$-partitions are the topic of [18, $\S 4.5]$, we will need to work in the slightly more general setting found in [17].

For our purposes, it is most convenient to say that a labelling $\omega$ of a poset $P$ is an injection $\omega: P \hookrightarrow\{1,2, \ldots\}$.

Definition 2.1. Let $P$ be a finite partially ordered set with a labelling $\omega$. A $(P, \omega)$-partition is a map $f: P \rightarrow\{0,1,2, \ldots\}$ with the following properties:

(i) $f$ is order-reversing: if $y \leq z$ in $P$ then $f(y) \geq f(z)$,

(ii) if $y<z$ in $P$ and $\omega(y)>\omega(z)$, then $f(y)>f(z)$.

In short, $(P, \omega)$-partitions are order-reversing maps with certain strictness conditions determined by $\omega$. We will denote the set of $(P, \omega)$-partitions by $\mathcal{A}(P, \omega)$.

Note. If $P$ is a poset with elements contained in the set $\{1,2, \ldots, n\}$, then in the labelled poset $(P, \omega)$, each vertex $i$ will have a label $\omega(i)$ associated to it and, in general, we certainly need not have $\omega(i)=i$. However, in our case, we will always take $\omega(i)=i$, since this is sufficient to yield the desired outcome.

Define the generating function $F(P, \omega ; \mathbf{x})$ in the variables $\mathbf{x}=\left(x_{1}, x_{2}, \ldots, x_{n}\right)$ by

$$
F(P, \omega ; \mathbf{x})=\sum_{f \in \mathcal{A}(P, \omega)}\left(\prod_{p \in P}\left(x_{\omega(p)}\right)^{f(p)}\right) .
$$

For any $n$-element poset $P$, by [17, Prop. 7.1] in the case when $\omega=$ id, the identity map, we have

$$
F(P, \omega ; \mathbf{x})=\sum_{\sigma \in L(P)} \frac{\prod_{j \in D(\sigma)} x_{\sigma(1)} x_{\sigma(2)} \ldots x_{\sigma(j)}}{\prod_{i=1}^{n}\left(1-x_{\sigma(1)} x_{\sigma(2)} \ldots x_{\sigma(i)}\right)} .
$$

Comparing this with (2.2), we deduce that

$$
\left\langle\kappa_{n}(\mathbf{q}), u ш v\right\rangle=\left(1-q_{1} q_{2} \cdots q_{n}\right) F\left(P_{u, v}, \mathrm{id}, \mathbf{q}\right) .
$$

The structure of $P_{u, v}$ is simple enough that we can actually get a nice expression for $F\left(P_{u, v}, \mathrm{id}, \mathbf{q}\right)$. Indeed, when $P$ is simply a total order with elements labelled $u(1), u(2), \ldots, u(r)$ from bottom to top, we see that

$$
F(P, \omega ; \mathbf{x})=\frac{\prod_{j \in D(u)} x_{u(1)} x_{u(2)} \ldots x_{u(j)}}{\prod_{i=1}^{r}\left(1-x_{u(1)} x_{u(2)} \ldots x_{u(i)}\right)},
$$


where $u$ is the word $u(1) u(2) \ldots u(r)$. The terms in the denominator ensure that the $(P, \omega)$-partitions are order-reversing, while the terms in the numerator take care of the strictness conditions. Furthermore, if $P$ is a disjoint union $P=P_{1}+P_{2}$, then let $\omega_{i}$ denote the labelling $\omega$ restricted to the elements of $P_{i}$, for $i=1,2$. We see that

$$
F(P, \omega ; \mathbf{x})=F\left(P_{1}, \omega_{1} ; \mathbf{x}\right) F\left(P_{2}, \omega_{2} ; \mathbf{x}\right)
$$

Combining (2.3) and (2.4), we deduce that

$$
F\left(P_{u, v}, \mathrm{id}, \mathbf{x}\right)=\frac{\left(\prod_{j \in D(u)} x_{u(1)} x_{u(2)} \cdots x_{u(j)}\right)\left(\prod_{\ell \in D(v)} x_{v(1)} x_{v(2)} \cdots x_{v(\ell)}\right)}{\prod_{i=1}^{r}\left(1-x_{u(1)} x_{u(2)} \cdots x_{u(i)}\right) \prod_{k=1}^{s}\left(1-x_{v(1)} x_{v(2)} \cdots x_{v(k)}\right)} .
$$

We finally conclude that

$$
\begin{aligned}
\left\langle\kappa_{n}(\mathbf{q}), u ш v\right\rangle & =\left(1-q_{1} \cdots q_{n}\right) F\left(P_{u, v}, \mathrm{id}, \mathbf{q}\right) \\
& =\left(1-q_{1} \cdots q_{n}\right) \frac{\left(\prod_{j \in D(u)} q_{u(1)} \cdots q_{u(j)}\right)\left(\prod_{\ell \in D(v)} q_{v(1)} \cdots q_{v(\ell)}\right)}{\left(\prod_{i=1}^{r} 1-q_{u(1)} \cdots q_{u(i)}\right)\left(\prod_{k=1}^{s} 1-q_{v(1)} \cdots q_{v(k)}\right)} \\
& =0,
\end{aligned}
$$

because of the conditions on $\mathbf{q}$ and because $r, s<n$. This yields Theorem 1.7.

\section{$3 \quad \kappa_{n}(\mathbf{q})$ is idempotent}

One way to show that the Klyachko idempotent is idempotent is to define an element $\eta_{n}$ of $K S_{n}$ such that

$$
\eta_{n} \kappa_{n}=\kappa_{n} \text { and } \kappa_{n} \eta_{n}=\eta_{n}
$$

(See [10], [16, Lemma 8.19].) Then it follows that

$$
\kappa_{n}^{2}=\kappa_{n} \eta_{n} \kappa_{n}=\eta_{n} \kappa_{n}=\kappa_{n}
$$

as required. Throughout, let $\gamma$ denote the $n$-cycle $(1,2, \ldots, n) \in S_{n}$. Let $\zeta$ denote the primitive $n$th root of unity from the definition of $\kappa_{n}$. Then a suitable element $\eta_{n}$ is given by

$$
\eta_{n}=\frac{1}{n} \sum_{i=0}^{n-1} \frac{\gamma^{i}}{\zeta^{i}}
$$

We wish to apply the same principle to show that $\kappa_{n}(\mathbf{q})$ is idempotent, thus proving Theorem 1.8. We define an element $\eta_{n}(\mathbf{q})$ of $K(\mathbf{q}) S_{n}$ by

$$
\eta_{n}(\mathbf{q})=\sum_{i=0}^{n-1} \operatorname{maj}_{\mathbf{q}}\left(\gamma^{i}\right) \gamma^{i}
$$


The reader is encouraged to check that if $q_{1}, \ldots, q_{n}$ are all mapped to $\zeta$, then $\eta_{n}(\mathbf{q})$ maps to $\eta_{n}$. Our goal, therefore, for the remainder of this section is to show that:

$$
\begin{aligned}
& \eta_{n}(\mathbf{q}) \ltimes \kappa_{n}(\mathbf{q})=\kappa_{n}(\mathbf{q}), \quad \text { and } \\
& \kappa_{n}(\mathbf{q}) \ltimes \eta_{n}(\mathbf{q})=\eta_{n}(\mathbf{q}) .
\end{aligned}
$$

Because we are taking twisted products, we will need to know how, for example, $\gamma\left[\operatorname{maj}_{\mathbf{q}}(\sigma)\right]$ compares to $\operatorname{maj}_{\mathbf{q}}(\sigma)$. For notational convenience, for any $\sigma \in S_{n}$, let us write $\operatorname{maj}_{\mathbf{q}}(\sigma)=\frac{N_{\mathbf{q}}(\sigma)}{D_{\mathbf{q}}(\sigma)}$, with

$$
\begin{aligned}
& N_{\mathbf{q}}(\sigma)=\prod_{j \in D(\sigma)} q_{\sigma(1)} q_{\sigma(2)} \ldots q_{\sigma(j)} \\
& D_{\mathbf{q}}(\sigma)=\prod_{i=1}^{n-1}\left(1-q_{\sigma(1)} q_{\sigma(2)} \ldots q_{\sigma(i)}\right) .
\end{aligned}
$$

("N" stands for numerator, and "D" for denominator.) The following result extends [15, Lemma 11].

Lemma 3.1. For all $\sigma, \tau \in S_{n}$, we have the following identities among elements of $K(\mathbf{q})$ :

$$
\gamma\left[N_{\mathbf{q}}(\sigma)\right]=q_{1} N_{\mathbf{q}}(\gamma \sigma)
$$

$$
\tau\left[D_{\mathbf{q}}(\sigma)\right]=D_{\mathbf{q}}(\tau \sigma)
$$

(iii)

$$
\gamma^{i}\left[\operatorname{maj}_{\mathbf{q}}(\sigma)\right]=q_{1} \cdots q_{i} \cdot \operatorname{maj}_{\mathbf{q}}\left(\gamma^{i} \sigma\right)
$$

$$
N_{\mathbf{q}}\left(\sigma \gamma^{i}\right)=\left(q_{\sigma(1)} \cdots q_{\sigma(i)}\right)^{-\bar{d}(\sigma)} N_{\mathbf{q}}(\sigma)
$$

Proof. (i) By definition,

$$
\begin{aligned}
\gamma\left[N_{\mathbf{q}}(\sigma)\right] & =\prod_{j \in D(\sigma)} q_{\gamma(\sigma(1))} \ldots q_{\gamma(\sigma(j))} \\
& =\prod_{j \in D(\sigma)} q_{(\gamma \sigma)(1)} \ldots q_{(\gamma \sigma)(j)}
\end{aligned}
$$

since $\gamma(\sigma(i))=(\gamma \sigma)(i)$.

The argument that follows is best understood by first trying some simple examples. If $\sigma(n)=n$, then $D(\gamma \sigma)=D(\sigma)+\{n-1\}$. Therefore,

$$
\begin{aligned}
N_{\mathbf{q}}(\gamma \sigma) & =\left(q_{\gamma \sigma(1)} q_{\gamma \sigma(2)} \cdots q_{\gamma \sigma(n-1)}\right) \gamma\left[N_{\mathbf{q}}(\sigma)\right] \\
& =\left(q_{2} q_{3} \cdots q_{n}\right) \gamma\left[N_{\mathbf{q}}(\sigma)\right] \\
& =\frac{\gamma\left[N_{\mathbf{q}}(\sigma)\right]}{q_{1}} .
\end{aligned}
$$


If $\sigma^{-1}(n)=i<n$, then $D(\gamma \sigma)=D(\sigma)+\{i-1\}-\{i\}$, where we set $\{0\}=\emptyset$. Therefore,

$$
N_{\mathbf{q}}(\gamma \sigma)=\frac{q_{\gamma \sigma(1)} \cdots q_{\gamma \sigma(i-1)}}{q_{\gamma \sigma(1)} \cdots q_{\gamma \sigma(i)}} \gamma\left[N_{\mathbf{q}}(\sigma)\right]=\frac{\gamma\left[N_{\mathbf{q}}(\sigma)\right]}{q_{1}} .
$$

(ii) This follows directly from the fact that $\tau(\sigma(i))=(\tau \sigma)(i)$.

(iii) By (i) and (ii), this is clearly true when $i=1$. Working by induction,

$$
\begin{aligned}
\gamma^{i}\left[\operatorname{maj}_{\mathbf{q}}(\sigma)\right] & =\gamma\left[\gamma^{i-1}\left[\operatorname{maj}_{\mathbf{q}}(\sigma)\right]\right] \\
& =\gamma\left[q_{1} \cdots q_{i-1} \cdot \operatorname{maj}_{\mathbf{q}}\left(\gamma^{i-1} \sigma\right)\right] \\
& =q_{2} \cdots q_{i} \cdot \gamma\left[\operatorname{maj}_{\mathbf{q}}\left(\gamma^{i-1} \sigma\right)\right] \\
& =q_{1} q_{2} \cdots q_{i} \cdot \operatorname{maj}_{\mathbf{q}}\left(\gamma^{i} \sigma\right) .
\end{aligned}
$$

(iv) We first show that

$$
N_{\mathbf{q}}(\sigma \gamma)=\frac{N_{\mathbf{q}}(\sigma)}{\left(q_{\sigma(1)}\right)^{\bar{d}(\sigma)}} .
$$

Indeed, suppose that $\sigma(1)>\sigma(n)$. Then

$$
N_{\mathbf{q}}(\sigma \gamma)=\frac{N_{\mathbf{q}}(\sigma)}{\left(q_{\sigma(1)}\right)^{d(\sigma)}}
$$

holds directly, and $d(\sigma)=\bar{d}(\sigma)$. If $\sigma(1)<\sigma(n)$, then

$$
\begin{aligned}
N_{\mathbf{q}}(\sigma \gamma) & =\frac{q_{\sigma(2)} \cdots q_{\sigma(n)} N_{\mathbf{q}}(\sigma)}{\left(q_{\sigma(1)}\right)^{d(\sigma)}} \\
& =\frac{N_{\mathbf{q}}(\sigma)}{\left(q_{\sigma(1)}\right)^{d(\sigma)+1}},
\end{aligned}
$$

and $d(\sigma)+1=\bar{d}(\sigma)$.

Proceeding by induction,

$$
\begin{aligned}
N_{\mathbf{q}}\left(\sigma \gamma^{i}\right) & =N_{\mathbf{q}}\left(\left(\sigma \gamma^{i-1}\right) \gamma\right) \\
& =\frac{N_{\mathbf{q}}\left(\sigma \gamma^{i-1}\right)}{\left(q_{\sigma \gamma^{i-1}(1)}\right)^{\bar{d}\left(\sigma \gamma^{i-1}\right)}} \\
& =\frac{N_{\mathbf{q}}\left(\sigma \gamma^{i-1}\right)}{\left(q_{\sigma(i)}\right)^{\bar{d}(\sigma)}} \\
& =\left(q_{\sigma(1)} \cdots q_{\sigma(i)}\right)^{-\bar{d}(\sigma)} N_{\mathbf{q}}(\sigma) .
\end{aligned}
$$

Before proving (3.1) and (3.2), we state one further necessary result, which is essentially taken word-for-word from [15]. As usual, $\delta_{i, j}$ denotes the Kronecker delta, defined to be 1 if $i=j$, and 0 otherwise. 
Proposition 3.2. [15, Corollary 10] Let $\alpha_{1}, \ldots, \alpha_{n}$ be elements of a field such that the product $\alpha_{1} \alpha_{2} \cdots \alpha_{n}$ is equal to 1 and each subproduct is different from 1 . For $k=0,1, \ldots, n-1$,

$$
\sum_{i=0}^{n-1} \frac{\left(\alpha_{i+1} \ldots \alpha_{n}\right)^{k}}{\left(1-\alpha_{i+1}\right)\left(1-\alpha_{i+1} \alpha_{i+2}\right) \cdots\left(1-\alpha_{i+1} \alpha_{i+2} \cdots \alpha_{i+n-1}\right)}=\delta_{0, k}
$$

where all subscripts on $\alpha$ are taken modulo $n$.

Here and henceforth, unless otherwise stated, all subscripts on $\mathbf{q}$ are taken modulo $n$. Proof of (3.1). We wish to show that

$$
\left(\sum_{i=0}^{n-1} \operatorname{maj}_{\mathbf{q}}\left(\gamma^{i}\right) \gamma^{i}\right) \ltimes\left(\sum_{\sigma \in S_{n}} \operatorname{maj}_{\mathbf{q}}(\sigma) \sigma\right)=\sum_{\tau \in S_{n}} \operatorname{maj}_{\mathbf{q}}(\tau) \tau .
$$

The left-hand side can be rewritten as

$$
\begin{aligned}
& \sum_{i, \sigma} \operatorname{maj}_{\mathbf{q}}\left(\gamma^{i}\right) \cdot \gamma^{i}\left[\operatorname{maj}_{\mathbf{q}}(\sigma)\right] \gamma^{i} \sigma \\
= & \sum_{i, \sigma} \operatorname{maj}_{\mathbf{q}}\left(\gamma^{i}\right) \cdot q_{1} \cdots q_{i} \cdot \operatorname{maj}_{\mathbf{q}}\left(\gamma^{i} \sigma\right) \gamma^{i} \sigma
\end{aligned}
$$

by Lemma 3.1(iii). Therefore, showing (3.4) is equivalent to showing that

$$
\sum_{\substack{i, \sigma \\ \gamma^{i} \sigma=\tau}} \operatorname{maj}_{\mathbf{q}}\left(\gamma^{i}\right) \cdot q_{1} \cdots q_{i} \cdot \operatorname{maj}_{\mathbf{q}}\left(\gamma^{i} \sigma\right)=\operatorname{maj}_{\mathbf{q}}(\tau)
$$

for all $\tau \in S_{n}$. Here, the left-hand side simplifies to

$$
\begin{aligned}
& \operatorname{maj}_{\mathbf{q}}(\tau) \sum_{\substack{i, \sigma \\
\gamma^{i} \sigma=\tau}} \operatorname{maj}_{\mathbf{q}}\left(\gamma^{i}\right) \cdot q_{1} \cdots q_{i} \\
= & \operatorname{maj}_{\mathbf{q}}(\tau) \sum_{i=0}^{n-1} \operatorname{maj}_{\mathbf{q}}\left(\gamma^{i}\right) \cdot q_{1} \cdots q_{i} \\
= & \operatorname{maj}_{\mathbf{q}}(\tau) \sum_{i=0}^{n-1} \frac{1}{\left(1-q_{i+1}\right)\left(1-q_{i+1} q_{i+2}\right) \cdots\left(1-q_{i+1} q_{i+2} \cdots q_{i+n-1}\right)}
\end{aligned}
$$

by Lemma 3.1(iii) with $\sigma=$ id or, alternatively, by direct calculation of maj $\mathbf{j}_{\mathbf{q}}\left(\gamma^{i}\right)$. Applying Proposition [3.2, we see that the expression of (3.5) equals $\operatorname{maj}_{\mathbf{q}}(\tau)$, as required.

Proof of (3.2). We wish to show that

$$
\left(\sum_{\sigma \in S_{n}} \operatorname{maj}_{\mathbf{q}}(\sigma) \sigma\right) \ltimes\left(\sum_{i=0}^{n-1} \operatorname{maj}_{\mathbf{q}}\left(\gamma^{i}\right) \gamma^{i}\right)=\sum_{j=0}^{n-1} \operatorname{maj}_{\mathbf{q}}\left(\gamma^{j}\right) \gamma^{j} .
$$


Since the left-hand side can be rewritten as

$$
\sum_{\sigma, i} \operatorname{maj}_{\mathbf{q}}(\sigma) \cdot \sigma\left[\operatorname{maj}_{\mathbf{q}}\left(\gamma^{i}\right)\right] \sigma \gamma^{i}
$$

showing (3.6) is equivalent to showing that

$$
\sum_{\substack{\sigma, i \\ \sigma \gamma^{i}=\tau}} \operatorname{maj}_{\mathbf{q}}(\sigma) \cdot \sigma\left[\operatorname{maj}_{\mathbf{q}}\left(\gamma^{i}\right)\right]= \begin{cases}\operatorname{maj}_{\mathbf{q}}\left(\gamma^{j}\right) & \text { if } \tau=\gamma^{j} \text { for some } j \\ 0 & \text { otherwise. }\end{cases}
$$

The left-hand side of (3.7) can be rewritten as

$$
\begin{aligned}
& \sum_{i=0}^{n-1} \operatorname{maj}_{\mathbf{q}}\left(\tau \gamma^{-i}\right) \cdot\left(\tau \gamma^{-i}\right)\left[\operatorname{maj}_{\mathbf{q}}\left(\gamma^{i}\right)\right] \\
= & \sum_{i=0}^{n-1} \operatorname{maj}_{\mathbf{q}}\left(\tau \gamma^{i}\right) \cdot\left(\tau \gamma^{i}\right)\left[\operatorname{maj}_{\mathbf{q}}\left(\gamma^{n-i}\right)\right] .
\end{aligned}
$$

By Lemma 3.1(iv),

$$
N_{\mathbf{q}}\left(\tau \gamma^{i}\right)=\left(q_{\tau(1)} \cdots q_{\tau(i)}\right)^{-\bar{d}(\tau)} N_{\mathbf{q}}(\tau)
$$

Also, we see directly that

$$
D_{\mathbf{q}}\left(\tau \gamma^{i}\right)=\left(1-q_{\tau(i+1)}\right)\left(1-q_{\tau(i+1)} q_{\tau(i+2)}\right) \cdots\left(1-q_{\tau(i+1)} q_{\tau(i+2)} \cdots q_{\tau(i+n-1)}\right) .
$$

As for the term $\left(\tau \gamma^{i}\right)\left[\operatorname{maj}_{\mathbf{q}}\left(\gamma^{n-i}\right)\right]$, we have that

$$
\begin{aligned}
\left(\tau \gamma^{i}\right)\left[N_{\mathbf{q}}\left(\gamma^{n-i}\right)\right] & =\left(\tau \gamma^{i}\right)\left[q_{n-i+1} \cdots q_{n}\right] \\
& =\tau\left[q_{1} \cdots q_{i}\right] \\
& =q_{\tau(1)} \cdots q_{\tau(i)},
\end{aligned}
$$

while, by Lemma 3.1 (ii), $\left(\tau \gamma^{i}\right)\left[D_{\mathbf{q}}\left(\gamma^{-i}\right)\right]=D_{\mathbf{q}}(\tau)$.

Putting this all together, we get that

$$
\begin{aligned}
& \sum_{\substack{\sigma, i \\
\sigma \gamma^{i}=\tau}} \operatorname{maj}_{\mathbf{q}}(\sigma) \cdot \sigma\left[\operatorname{maj}_{\mathbf{q}}\left(\gamma^{i}\right)\right] \\
= & \frac{N_{\mathbf{q}}(\tau)}{D_{\mathbf{q}}(\tau)} \sum_{i=0}^{n-1} \frac{\left(q_{\tau(1)} \cdots q_{\tau(i)}\right)^{1-\bar{d}(\tau)}}{\left(1-q_{\tau(i+1)}\right) \cdots\left(1-q_{\tau(i+1)} q_{\tau(i+2)} \cdots q_{\tau(i+n-1)}\right)} \\
= & \frac{N_{\mathbf{q}}(\tau)}{D_{\mathbf{q}}(\tau)} \sum_{i=0}^{n-1} \frac{\left(q_{\tau(i+1)} \cdots q_{\tau(n)}\right)^{\bar{d}(\tau)-1}}{\left(1-q_{\tau(i+1)}\right) \cdots\left(1-q_{\tau(i+1)} q_{\tau(i+2)} \cdots q_{\tau(i+n-1)}\right)} .
\end{aligned}
$$

Since $\bar{d}(\tau)=1$ if $\tau=\gamma^{j}$ for some $j$, and $2 \leq \bar{d}(\tau) \leq n-1$ otherwise, applying Proposition 3.2 gives exactly the desired equality (3.7). 


\section{$4 \kappa_{n}(\mathbf{q})$ generates the multilinear part of the free Lie algebra}

As before, let $\mathcal{L}_{n}\left(\operatorname{resp} . \mathcal{L}_{n}(\mathbf{q})\right)$ denote the set of Lie elements in $K S_{n}\left(\operatorname{resp} . K(\mathbf{q}) S_{n}\right)$. Our goal for this section is to show that

$$
K(\mathbf{q}) S_{n} \ltimes \kappa_{n}(\mathbf{q})=\mathcal{L}_{n}(\mathbf{q}) .
$$

By Theorem 1.7, we know that $\kappa_{n}(\mathbf{q}) \in \mathcal{L}_{n}(\mathbf{q})$, while (1.1) states that $\mathcal{L}_{n}(\mathbf{q})$ is a left ideal of $K(\mathbf{q}) S_{n}$. Hence, we have that $K(\mathbf{q}) S_{n} \ltimes \kappa_{n}(\mathbf{q}) \subseteq \mathcal{L}_{n}(\mathbf{q})$. Also, it is well-known that $\mathcal{L}_{n}$ has dimension $(n-1)$ !, from which it follows that $\mathcal{L}_{n}(\mathbf{q})$ has dimension $(n-1)$ !. Therefore, it suffices to show that $K(\mathbf{q}) S_{n} \ltimes \kappa_{n}(\mathbf{q})$ has dimension at least $(n-1)$ !.

As we saw in Section 3, we have the following two identities:

$$
\begin{aligned}
& \eta_{n}(\mathbf{q}) \ltimes \kappa_{n}(\mathbf{q})=\kappa_{n}(\mathbf{q}), \\
& \kappa_{n}(\mathbf{q}) \ltimes \eta_{n}(\mathbf{q})=\eta_{n}(\mathbf{q}) .
\end{aligned}
$$

We claim that these identities can be used to give a bijection $\phi$ from $K(\mathbf{q}) S_{n} \ltimes \kappa_{n}(\mathbf{q})$ to $K(\mathbf{q}) S_{n} \ltimes \eta_{n}(\mathbf{q})$. Indeed, for $x \in K(\mathbf{q}) S_{n} \ltimes \kappa_{n}(\mathbf{q})$, let

$$
\phi(x)=x \ltimes \eta_{n}(\mathbf{q}) .
$$

Define a map $\psi: K(\mathbf{q}) S_{n} \ltimes \eta_{n}(\mathbf{q}) \rightarrow K(\mathbf{q}) S_{n} \ltimes \kappa_{n}(\mathbf{q})$ by

$$
\psi(y)=y \ltimes \kappa_{n}(\mathbf{q})
$$

for any $y \in K(\mathbf{q}) S_{n} \ltimes \eta_{n}(\mathbf{q})$. For $x \in K(\mathbf{q}) S_{n} \ltimes \kappa_{n}(\mathbf{q})$, we know that $x=x^{\prime} \ltimes \kappa_{n}(\mathbf{q})$ for some $x^{\prime} \in K(\mathbf{q}) S_{n}$. Therefore,

$$
\begin{aligned}
\psi(\phi(x)) & =\psi\left(x^{\prime} \ltimes \kappa_{n}(\mathbf{q}) \ltimes \eta_{n}(\mathbf{q})\right) \\
& =\psi\left(x^{\prime} \ltimes \eta_{n}(\mathbf{q})\right) \\
& =\left(x^{\prime} \ltimes \eta_{n}(\mathbf{q})\right) \ltimes \kappa_{n}(\mathbf{q}) \\
& =x^{\prime} \ltimes \kappa_{n}(\mathbf{q}) \\
& =x .
\end{aligned}
$$

Similarly, $\phi(\psi(y))=y$ for any $y \in K(\mathbf{q}) S_{n} \ltimes \eta_{n}(\mathbf{q})$, and so $\phi$ is a bijection. We conclude that to prove Theorem 1.9, it remains to show that $K(\mathbf{q}) S_{n} \ltimes \eta_{n}(\mathbf{q})$ has dimension at least $(n-1)$ !.

Consider the set $B=\left\{\sigma \ltimes \eta_{n}(\mathbf{q}) \mid \sigma \in S_{n}, \sigma(1)=1\right\}$. Clearly, $B \subseteq K(\mathbf{q}) S_{n} \ltimes \eta_{n}(\mathbf{q})$. We claim that $B$ forms a basis of $K(\mathbf{q}) S_{n} \ltimes \eta_{n}(\mathbf{q})$. Suppose $\sigma$ and $\tau$ are distinct permutations with $\sigma(1)=\tau(1)=1$. Since

$$
\sigma \ltimes \eta_{n}(\mathbf{q})=\sum_{i=0}^{n-1} \sigma\left[\operatorname{maj}_{\mathbf{q}}\left(\gamma^{i}\right)\right] \sigma \gamma^{i}
$$


we see that $\sigma \ltimes \eta_{n}(\mathbf{q})$ is a linear combination of permutations of the form $\sigma \gamma^{i}$. Similarly, $\tau \ltimes \eta_{n}(\mathbf{q})$ is a linear combination of permutations of the form $\tau \gamma^{j}$. But since $\sigma$ and $\tau$ are distinct and $\sigma(1)=\tau(1)=1$, there do not exist $k, \ell$ such that $\sigma \gamma^{k}=\tau \gamma^{\ell}$. It follows that $\sigma \ltimes \eta_{n}(\mathbf{q}) \neq \tau \ltimes \eta_{n}(\mathbf{q})$ and, furthermore, that the elements of $B$ are linearly independent. We conclude that $B$ consists of $(n-1)$ ! linearly independent elements, and so $K(\mathbf{q}) S_{n} \ltimes \eta_{n}(\mathbf{q})$ has dimension at least $(n-1)$ !, thus proving Theorem 1.9.

We will conclude by showing independently that $B$ spans $K(\mathbf{q}) S_{n} \ltimes \eta_{n}(\mathbf{q})$, thereby reproving that the dimension of $K(\mathbf{q}) S_{n} \ltimes \eta_{n}(\mathbf{q})$, and hence of $K(\mathbf{q}) S_{n} \ltimes \kappa_{n}(\mathbf{q})$, is $(n-1)$ !.

Every permutation $\tau \in S_{n}$ is of the form $\sigma \gamma^{j}$ for some $\sigma$ with $\sigma(1)=1$. We claim that $\tau \ltimes \eta_{n}(\mathbf{q})$ is then simply a scalar multiple of $\sigma \ltimes \eta_{n}(\mathbf{q}) \in B$. Indeed, using Lemma 3.1(iii), we have

$$
\begin{aligned}
\tau \ltimes \eta_{n}(\mathbf{q}) & =\left(\sigma \gamma^{j}\right) \ltimes \sum_{i=0}^{n-1} \operatorname{maj}_{\mathbf{q}}\left(\gamma^{i}\right) \gamma^{i} \\
& =\sum_{i=0}^{n-1}\left(\sigma \gamma^{j}\right)\left[\operatorname{maj}_{\mathbf{q}}\left(\gamma^{i}\right)\right] \sigma \gamma^{j+i} \\
& =\sum_{i=0}^{n-1} q_{\sigma(1)} \cdots q_{\sigma(j)} \cdot \sigma\left[\operatorname{maj}_{\mathbf{q}}\left(\gamma^{j+i}\right)\right] \sigma \gamma^{j+i} \\
& =q_{\sigma(1)} \cdots q_{\sigma(j)} \sum_{i=0}^{n-1} \sigma\left[\operatorname{maj}_{\mathbf{q}}\left(\gamma^{i}\right)\right] \sigma \gamma^{i} \\
& =q_{\sigma(1)} \cdots q_{\sigma(j)} \cdot \sigma \ltimes \eta_{n}(\mathbf{q}) .
\end{aligned}
$$

It follows that every element of $K(\mathbf{q}) S_{n} \ltimes \eta_{n}(\mathbf{q})$ can be written as a linear combination of elements of $B$, as required.

\section{$5 \quad$ Infinite product expansion}

Let $\mathbf{x}=x_{1}, x_{2}, \ldots$ be an infinite sequence of variables in a field $K$ of characteristic 0 . In effect, the $x_{i}$ 's will play the role formerly played by the $q_{i}$ 's. We switch variables to emphasize two key differences with the material in this section. The first is that $n$ will no longer be fixed and the second is that there will be no restriction on the $x_{i}$ 's analogous to the restriction $q_{1} \cdots q_{n}=1$ on the $q_{i}$ 's. We let $K\left[\left[x_{1}, \ldots, x_{n}\right]\right]$ denote the algebra of formal power series in $x_{1}, \ldots, x_{n}$ over the field $K$.

The central object of study in this section will be $\Theta(\mathbf{x})$, which is an element of $\oplus_{n \geq 0} K\left[\left[x_{1}, \ldots, x_{n}\right]\right] S_{n}$, and is defined as follows:

$$
\begin{aligned}
\Theta(\mathbf{x}) & =\sum_{n \geq 0} \sum_{\sigma \in S_{n}} \frac{\prod_{j \in D(\sigma)} x_{\sigma(1)} x_{\sigma(2)} \ldots x_{\sigma(j)}}{\prod_{i=1}^{n}\left(1-x_{\sigma(1)} x_{\sigma(2)} \ldots x_{\sigma(i)}\right)} \sigma \\
& =\sum_{n \geq 0} \frac{\kappa_{n}\left(x_{1}, \ldots, x_{n}\right)}{\left(1-x_{1} \cdots x_{n}\right)} .
\end{aligned}
$$


We can think of $\Theta(\mathbf{x})$ as a generating function for $\kappa_{n}\left(x_{1}, \ldots, x_{n}\right)$ and, in particular, we see that knowing $\Theta(\mathbf{x})$ allows us to extract $\kappa_{n}(\mathbf{q})$, for any $n$.

Our goal for this section is to show that $\Theta(\mathbf{x})$ can be expressed as a simple infinite product. To do this, we first need to put an appropriate ring structure on $\oplus_{n \geq 0} K\left[\left[x_{1}, \ldots, x_{n}\right]\right] S_{n}$. As we shall see, once we have done this correctly, we will have completed much of the work necessary to fulfill our goal.

First, we will recall from [14] the associative product $\star$ defined on $\oplus_{n \geq 0} K S_{n}$. We let $\mathbb{P}$ denote the positive integers, and we will be working with words on the alphabet $\mathbb{P}$. If $u$ and $v$ are two such words, then we define the product $u \cdot v$ to be simply their concatenation. If $w=w(1) \ldots w(n)$ and the letters $w(1), \ldots, w(n)$ of $w$ are distinct, then we define the standardization of $w$ to be the unique permutation $\operatorname{st}(w)$ of $S_{n}$ satisfying

$$
\operatorname{st}(w)(i) \leq \operatorname{st}(w)(j) \Leftrightarrow w(i) \leq w(j)
$$

for all $1 \leq i, j \leq n$. For example, $\operatorname{st}(5716)=2413$. If $\sigma \in S_{s}$ and $\tau \in S_{t}$ then we define $\star$ by

$$
\sigma \star \tau=\sum_{u, v} u \cdot v
$$

where the sum is over all $u, v$ such that $u \cdot v \in S_{s+t}$ with $\operatorname{st}(u)=\sigma$ and $\operatorname{st}(v)=\tau$. For example, $132 \star 1=1324+1423+1432+2431$. Extending by linearity gives a product on $\oplus_{n \geq 0} K S_{n}$. Since $\sigma \star \tau$ is a multiplicity-free sum, we can write $w \in \sigma \star \tau$ to mean that $w$ appears as a term in $\sigma \star \tau$.

Before extending $\star$ to $\oplus_{n \geq 0} K\left[\left[x_{1}, \ldots, x_{n}\right]\right] S_{n}$, we will introduce some convenient notation. If $f\left(x_{1}, \ldots, x_{n}\right) \in K\left[\left[x_{1}, \ldots, x_{n}\right]\right]$ and if $w$ is a word on $\mathbb{P}$ of length $n$ with distinct letters $a_{1}<a_{2}<\cdots<a_{n}$, then define $f(w)$ to be $f\left(x_{a_{1}}, \ldots, x_{a_{n}}\right)$. For example, if $f\left(x_{1}, x_{2}, x_{3}\right) \in K\left[\left[x_{1}, x_{2}, x_{3}\right]\right]$, then $f(382)=f\left(x_{2}, x_{3}, x_{8}\right)$. For $\sigma \in S_{s}$ and $\tau \in S_{t}$, we can then define

$$
\left(f\left(x_{1}, \ldots, x_{s}\right) \sigma\right) \star\left(g\left(x_{1}, \ldots, x_{t}\right) \tau\right)=\sum_{w \in \sigma \star \tau} f(w(1) \ldots w(s)) \cdot g(w(s+1) \ldots w(s+t)) w,
$$

and extend to $\oplus_{n \geq 0} K\left[\left[x_{1}, \ldots, x_{n}\right]\right] S_{n}$ by linearity. For example,

$$
\begin{aligned}
\left(x_{1}^{2} x_{2}^{2} x_{3} 132\right) \star\left(x_{1}^{3} 1\right)= & x_{1}^{2} x_{2}^{2} x_{3} x_{4}^{3} 1324+x_{1}^{2} x_{2}^{2} x_{4} x_{3}^{3} 1423 \\
& +x_{1}^{2} x_{3}^{2} x_{4} x_{2}^{3} 1432+x_{2}^{2} x_{3}^{2} x_{4} x_{1}^{3} 2431 .
\end{aligned}
$$

We see that the product $\star$ on $\oplus_{n \geq 0} K\left[\left[x_{1}, \ldots, x_{n}\right]\right] S_{n}$ is a natural extension of the $\oplus_{n \geq 0} K S_{n}$ version.

Remark 5.1. The product $\star$ on $\oplus_{n \geq 0} K\left[\left[x_{1}, \ldots, x_{n}\right]\right] S_{n}$ makes it an associative algebra. This follows from [1, §1.2]: our algebra is the completion, in an appropriate sense, of the algebra $\oplus_{n \geq 0} K\left[x_{1}, \ldots, x_{n}\right] S_{n}$; the latter is isomorphic to the $\mathscr{F}(V)$ of Baumann and Hohlweg when $V$ is taken to be the free $K$-module with basis $\mathbb{N}$ (the non-negative integers). Indeed, a word $i_{1} \ldots i_{n}$ on $\mathbb{N}$ corresponds to the monomial $x_{1}^{i_{1}} \cdots x_{n}^{i_{n}}$ in $K\left[\left[x_{1}, \ldots, x_{n}\right]\right]$. It also follows from [1] that $\oplus_{n \geq 0} K\left[\left[x_{1}, \ldots, x_{n}\right]\right] S_{n}$ can even be endowed with a Hopf algebra structure. 
We are almost ready to state the main result of this section. We let $\varepsilon$ denote the unique element of the symmetric group $S_{0}$. When expressing an infinite product of elements of $\oplus_{n \geq 0} K\left[\left[x_{1}, \ldots, x_{n}\right]\right] S_{n}$, we will use an arrow pointing left above the product symbol to denote that the product should be expanded from right to left. For example,

$$
\overleftarrow{\prod_{n \geq 0}} F(n)=\cdots \star F(2) \star F(1) \star F(0)
$$

The following result extends [8, Proposition 5.10].

Theorem 5.2. The generating function $\Theta(\mathbf{x})$ of $\kappa_{n}\left(x_{1}, \ldots, x_{n}\right)$ satisfies

$$
\Theta(\mathbf{x})=\overleftarrow{\prod_{n \geq 0}}\left(\varepsilon+x_{1}^{n} 1+x_{1}^{n} x_{2}^{n} 12+x_{1}^{n} x_{2}^{n} x_{3}^{n} 123+\cdots\right)
$$

The simplicity of (5.2) perhaps helps to explain why $\kappa_{n}(\mathbf{q})$ would have many nice properties, such as those from the earlier sections. We remark that each factor in the product, and the product itself, is a group-like element for the Hopf algebra structure of Remark 5.1. This is similar to the situation in [8] and is easily verified.

Proof. We can expand the right-hand side of (5.2) into nested sums as follows:

$$
\begin{aligned}
& \overleftarrow{n \geq 0}\left(\varepsilon+x_{1}^{n} 1+x_{1}^{n} x_{2}^{n} 12+x_{1}^{n} x_{2}^{n} x_{3}^{n} 123+\cdots\right) \\
= & \sum_{k \geq 0} \sum_{n_{1}>\cdots>n_{k} \geq 0} \sum_{a_{1}, \ldots, a_{k} \geq 1}\left(x_{1}^{n_{1}} \cdots x_{a_{1}}^{n_{1}} 12 \cdots a_{1}\right) \star \cdots \star\left(x_{1}^{n_{k}} \cdots x_{a_{k}}^{n_{k}} 12 \cdots a_{k}\right) \\
= & \sum_{n \geq 0} \sum_{\sigma \in S_{n}} \sum_{k \geq 0} \sum_{\sigma=u_{1} \cdots u_{k}}\left(x_{u_{1}}\right)^{n_{1}} \cdots\left(x_{u_{k}}\right)^{n_{k}} \sigma . \cdots n_{k} \geq 0
\end{aligned}
$$

where the fourth sum is over all non-empty increasing words $u_{1}, \ldots, u_{k}$ in $\mathbb{P}$ whose concatenation is the word $\sigma$, and where $x_{u}$ for the word $u=u(1) \ldots u(s)$ denotes $x_{u(1)} \cdots x_{u(s)}$.

We see that the coefficient $C(\sigma)$ of a fixed $\sigma \in S_{n}$ is then given by

$$
C(\sigma)=\sum_{k \geq 0} \sum_{\sigma=u_{1} \cdots u_{k}} \sum_{n_{1}>\cdots>n_{k} \geq 0}\left(x_{u_{1}}\right)^{n_{1}} \cdots\left(x_{u_{k}}\right)^{n_{k}},
$$

where the $u_{i}$ 's are increasing non-empty words. It will be helpful to rewrite $C(\sigma)$ as

$$
\begin{aligned}
C(\sigma) & =\sum_{k \geq 0} \sum_{\sigma=u_{1} \cdots u_{k}} \sum_{\substack{n_{1}>\cdots>n_{k} \geq 0\\
}}\left(x_{u_{1}}\right)^{n_{1}-n_{2}}\left(x_{u_{1} u_{2}}\right)^{n_{2}-n_{3}} \cdots\left(x_{u_{1} \ldots u_{k}}\right)^{n_{k}} \\
& =\sum_{k \geq 0} \sum_{\substack { \sigma=u_{1} \cdots u_{k} \\
\begin{subarray}{c}{m_{1}, \ldots, m_{k-1}>0 \\
m_{k} \geq 0{ \sigma = u _ { 1 } \cdots u _ { k } \\
\begin{subarray} { c } { m _ { 1 } , \ldots , m _ { k - 1 } > 0 \\
m _ { k } \geq 0 } }\end{subarray}}\left(x_{u_{1}}\right)^{m_{1}}\left(x_{u_{1} u_{2}}\right)^{m_{2}} \cdots\left(x_{u_{1} \ldots u_{k}}\right)^{m_{k}} .
\end{aligned}
$$

The condition that the $u_{i}$ 's be increasing words implies that $C(\sigma)$ can be restructured as

$$
C(\sigma)=\sum_{\substack{p_{1}, p_{2} \ldots, p_{n} \geq 0 \\ i \in D(\sigma) \Rightarrow p_{i}>0}}\left(x_{\sigma(1)}\right)^{p_{1}}\left(x_{\sigma(1)} x_{\sigma(2)}\right)^{p_{2}} \cdots\left(x_{\sigma(1)} \cdots x_{\sigma(n)}\right)^{p_{n}} .
$$


Observing that each monomial in the sum has $\prod_{j \in D(\sigma)} x_{\sigma(1)} x_{\sigma(2)} \ldots x_{\sigma(j)}$ as a factor, we get

$$
\begin{aligned}
C(\sigma)= & \left(\prod_{j \in D(\sigma)} x_{\sigma(1)} x_{\sigma(2)} \ldots x_{\sigma(j)}\right) \times \\
& \sum_{p_{1}, p_{2} \ldots, p_{n} \geq 0}\left(x_{\sigma(1)}\right)^{p_{1}}\left(x_{\sigma(1)} x_{\sigma(2)}\right)^{p_{2}} \cdots\left(x_{\sigma(1)} \cdots x_{\sigma(n)}\right)^{p_{n}} \\
= & \frac{\prod_{j \in D(\sigma)} x_{\sigma(1)} x_{\sigma(2)} \ldots x_{\sigma(j)}}{\prod_{i=1}^{n}\left(1-x_{\sigma(1)} x_{\sigma(2)} \ldots x_{\sigma(i)}\right)}
\end{aligned}
$$

as required.

We close with a remark that again relates our work to Stanley's $P$-partitions. Comparing (5.4) with [18, Lemma 4.5.2(a)], we see that $C(\sigma)$ is exactly the generating function for the set of all $\sigma$-compatible permutations, as defined in [18].

\section{Acknowledgements}

We thank François Bergeron for suggesting that we look for an infinite product expansion generalizing [8, Proposition 5.10], and Pierre Baumann for showing us the connection between $\oplus_{n \geq 0} K\left[\left[x_{1}, \ldots, x_{n}\right]\right] S_{n}$ and the $\mathscr{F}(V)$ of [1], as discussed in Remark 5.1. Also, we are grateful for the referee's careful reading and suggestions.

\section{References}

[1] Pierre Baumann and Christophe Hohlweg. A Solomon descent theory for the wreath products $G<\mathfrak{S}_{n}$. Preprint. http://www.arxiv.org/abs/math.C0/0503011, 2005.

[2] F. Bergeron, N. Bergeron, and A. M. Garsia. Idempotents for the free Lie algebra and q-enumeration. In Invariant theory and tableaux (Minneapolis, MN, 1988), volume 19 of IMA Vol. Math. Appl., pages 166-190. Springer, New York, 1990.

[3] Charles W. Curtis and Irving Reiner. Methods of representation theory. Vol. I. John Wiley \& Sons Inc., New York, 1981. With applications to finite groups and orders, Pure and Applied Mathematics, A Wiley-Interscience Publication.

[4] Gérard Duchamp, Daniel Krob, Bernard Leclerc, and Jean-Yves Thibon. Déformations de projecteurs de Lie. C. R. Acad. Sci. Paris Sér. I Math., 319(9):909914, 1994.

[5] Adriano M. Garsia. Combinatorial methods in the theory of Cohen-Macaulay rings. Adv. in Math., 38(3):229-266, 1980. 
[6] Adriano M. Garsia. Combinatorics of the free Lie algebra and the symmetric group. In Analysis, et cetera, pages 309-382. Academic Press, Boston, MA, 1990.

[7] A. M. Garsia and C. Reutenauer. A decomposition of Solomon's descent algebra. Adv. Math., 77(2):189-262, 1989.

[8] Israel M. Gelfand, Daniel Krob, Alain Lascoux, Bernard Leclerc, Vladimir S. Retakh, and Jean-Yves Thibon. Noncommutative symmetric functions. Adv. Math., 112(2):218-348, 1995.

[9] Florent Hivert. Hecke algebras, difference operators, and quasi-symmetric functions. Adv. Math., 155(2):181-238, 2000.

[10] A. A. Kljačko. Lie elements in a tensor algebra. Sibirsk. Mat. Ž., 15:1296-1304, 1430, 1974.

[11] D. Krob, B. Leclerc, and J.-Y. Thibon. Noncommutative symmetric functions. II. Transformations of alphabets. Internat. J. Algebra Comput., 7(2):181-264, 1997.

[12] Alain Lascoux, Bernard Leclerc, and Jean-Yves Thibon. Crystal graphs and qanalogues of weight multiplicities for the root system $A_{n}$. Lett. Math. Phys., 35(4):359-374, 1995.

[13] M. Lothaire. Combinatorics on words, volume 17 of Encyclopedia of Mathematics and its Applications. Addison-Wesley Publishing Co., Reading, Mass., 1983. A collective work by Dominique Perrin, Jean Berstel, Christian Choffrut, Robert Cori, Dominique Foata, Jean Eric Pin, Guiseppe Pirillo, Christophe Reutenauer, MarcelP. Schützenberger, Jacques Sakarovitch and Imre Simon, With a foreword by Roger Lyndon, Edited and with a preface by Perrin.

[14] Claudia Malvenuto and Christophe Reutenauer. Duality between quasi-symmetric functions and the Solomon descent algebra. J. Algebra, 177(3):967-982, 1995.

[15] Frédéric Patras and Christophe Reutenauer. On Dynkin and Klyachko idempotents in graded bialgebras. Adv. in Appl. Math., 28(3-4):560-579, 2002. Special issue in memory of Rodica Simion.

[16] Christophe Reutenauer. Free Lie algebras, volume 7 of London Mathematical Society Monographs. New Series. The Clarendon Press Oxford University Press, New York, 1993. Oxford Science Publications.

[17] Richard P. Stanley. Ordered structures and partitions. American Mathematical Society, Providence, R.I., 1972. Memoirs of the American Mathematical Society, No. 119.

[18] Richard P. Stanley. Enumerative combinatorics. Vol. I. Wadsworth \& Brooks/Cole Advanced Books \& Software, Monterey, CA, 1986. Second printing, Cambridge University Press, Cambridge/New York, 1997. 